\title{
Utilizing Sound Effects in Mobile User Interface Design
}

\author{
Hannu Korhonen, Jukka Holm, and Mikko Heikkinen \\ Nokia Research Center, P.O. Box 100, 33720 Tampere, Finland \\ \{hannu.j.korhonen, jukka.a.holm, mikko.o.heikkinen\}@nokia.com
}

\begin{abstract}
The current generation of mobile devices is capable of producing polyphonic sounds, has enough processing power for real-time signal processing, and much better sound quality than their predecessors. The importance of audio is increasing as we are moving towards multimodal user interfaces where audio is one of the major components. In this paper, we present new ways of using audio feedback more efficiently and intelligently in mobile user interfaces by utilizing real-time signal processing. To test the ideas in practice, a prototype calendar application was implemented. We arranged a one week field trial to validate the design ideas. The results indicate that sound effects are capable of passing information to the user in some extent, but they are more useful in impressing the user and making existing audio feedback sound better.
\end{abstract}

Keywords: Auditory interfaces, multi-modal interfaces, sonification, data auralization, mobile phones, calendar, non-speech audio, reverb, navigation, sound effects.

\section{Introduction}

Audio enhanced user interfaces have been studied for a long time. Despite this, the use of audio in UIs has not changed much and sounds have only been used for giving audio cues or simple musical tones. The current generation of mobile devices is capable of producing polyphonic sounds and has enough processing power for realtime signal processing. In addition, the devices have much better sound quality than their predecessors. Audio could be a very important element in user interfaces that can provide information for a user, give appropriate feedback on events and actions, and amuse the user.

In the 80's the main research question was how a large amount of data can be presented using audio [12]. Brown et al. demonstrated that auditory information could be used as effectively as visual information for a visual search task when speed is not a crucial issue [14]. Visual presentation of graphs was replaced with audio for data such as stock market data, economic indicators and other data [13]. Audio was also used for presenting different measurement results like infrared spectrograms and DNA sequence representations.

After the 80's, the research has extended to cover also new audio widgets, sound effects, auditory icons [3], earcons [4], as well as navigation and feedback in user interfaces. Larsson et al. have described how audio can be used in the context of 
virtual environments [11]. However, their findings can be generalized to cover other types of audio UIs as well. According to Larsson, audio can be used to give feedback to users' actions, carry information, provide information beyond the field of view, enhance visual representation, and immerse users to the environment. In addition to the non-speech sounds, also voice can be used as an output modality. Mainly this concerns speech interfaces, in which the user can hear information given by speech.

Giving feedback to user's actions is probably the most common way of using audio in user interfaces. The user can hear clicks or opening or closing sounds when they interact with the user interface widgets. Audio can also be used to replace elements in the visual modality. As Megan [14] points it out, "Auditory information can be used to describe certain attributes of visual displays. Not only may this combination reduce the visual workload, but it may also free screen real estate for other uses." In addition to replacing visual information on the screen, sounds can be used to auralize events outside of the current view (see e.g. Gaver's classic Arkola paper [10]).

Audio feedback can also support the visual representation of an UI and strengthen the emotion that the UI creates. For example, if the user interface uses cold colors and metallic or sharp audio, the resulting UI can be perceived as industrial.

One way of increasing user's immersion to an application is that user interface objects produce realistic sounds. The sound can be used for providing information about the physical dimensions of an object or mimic sounds that are heard when some objects are manipulated. An example on how manipulation and physical dimension sounds can be combined is when the user copies a file to another location. Moving the file on the screen produces a dragging sound. When the copying is ready, a dropping sound is heard, but the sound depends on the size of the file. Bigger file produces a heavier sound. [3]

The traditional mobile phone UI sounds can be divided into two categories: Alerting and feedback sounds. Alerting sounds are played when there is an incoming call, the battery is running out, or there is a pre-set calendar event. Feedback sounds are played in response to some user action, and they include warning and keypad tones.

One of the major challenges with mobile phone UI sounds is the context where the devices are used, since it is more diverse than with stationary devices. According to our experience, many people find it embarrasing or disturbing, if their mobile phone makes sounds in a public place. Therefore, mobile phone users tend to turn off all sounds or at least lower the volume level. One of the reasons for this behavior is that users do not think that current sounds are useful. A good example of this is the keypad tones. If the sounds do not supply information that the user really needs, they serve no real purpose. Also, if the sounds are too distinctive or unpleasant they are usually not tolerated. These issues have to obviously be taken into account when thinking about new ways of using audio in mobile UIs.

Another major problem has been the sound quality. The older phones were only capable of producing monotonic, monophonic buzzer sounds that were not very pleasing to the ear. Fortunately, with the current generation of phones, the sound quality has become much better and the sounds can be of almost any type: monophonic or polyphonic MIDI tones, MP3 files, Wave files, speech synthesis, and so on. The phones have enough processing power to support various sound effects such as reverb, delay, or different kinds of filters. 
This paper presents a study utilising UI sounds in mobile phones in new ways. The objective of the study was to provide design ideas and proposals on how audio feedback could be utilized more efficiently and intelligently in the mobile devices. Mobile devices are used in different contexts and situational impairment ${ }^{1}$ will make the user of visual modality sometimes very difficult or even impossible. Audio modality can enhance device usability and provide an alternative output modality in order to overcome the problems and inconveniences caused by situational impairment.

\section{Background}

In this section, we describe some relevant background information on earcons, auditory icons, and sonification in PC and mobile phone environments. The basic theory for sound effects that were used in our study is also covered.

\subsection{Earcons, Auditory Icons, and Sonification}

Using non-speech sounds to convey information in a user interface has been explored quite a lot in recent years. Previous studies can be roughly divided into three categories: Earcons, auditory icons, and sonification i.e. data auralization. So far most of them have concentrated on the PC environment, but the usage of sounds in mobile devices is also being studied increasingly.

Sonification refers to translating visual or other non-auditory events into sound. In [1], it is defined as "the use of nonspeech audio to convey information. More specifically, sonification is the transformation of data relations into perceived relations in an acoustic signal for the purposes of facilitating communication or interpretation." In many research papers, the sonified data has been complex and high-dimensional such as stock market information [15], economic indicators, earthquake data [16], weather reports [2], or health care [19].

Auditory icons and earcons represent two different strategies for using sound to represent actions and objects within an interface. Auditory icons are based on the use of natural, everyday sounds. In [3], Gaver defines them as "environmental sounds designed to be appropriate for the virtual environment of the interface." They are like sound effects for the computer: "For instance, selecting a file icon in a graphical user interface might make the sound of a notebook being tapped, with the type of file indicated by the material of the object, and the file size by the size of the struck object." Auditory icons have an intuitive link to the object or action that they represent.

Earcons, on the other hand, rely on the use of musical sounds in presenting information to a user. According to Brewster [4], they are "abstract, synthetic tones that can be used in structured combinations to create sound message to represent parts of an interface... Earcons are composed of motives, which are short, rhythmic

${ }^{1}$ Situational impairment is a moment in which the user is temporarily unable to use visual modality and normal input methods. The impairment can be caused by environment context elements like bright sunlight or cold weather, or it can be caused by specific task context (e.g. driving a car) or social context (disturbance caused to other persons in the vicinity). 
sequences of pitches with variable intensity, timbre and register." Earcons can be combined in various ways to produce complex audio messages, and are mostly used for supporting navigation in the menu hierarchy.

When comparing these two strategies, Gaver [3] states that "Auditory icons are designed to be easy to understand, but this may require exacting sound design. Earcons must be learned, but they are easy to create and manipulate especially using MIDI equipment... Auditory icons tend to be judged as mapping better (more clearly, more memorably) to interface events, but earcons tend to be judged as more pleasant."

While the results of using earcons in the PC environment have been positive (see e.g. [4] or [5]), they have not really taken off in the mobile world. The research results have been varying depending on the study. As an example, in [6] the authors have implemented a computer-based simulation of Nokia 6110 mobile phone to evaluate the benefit of earcons. The results showed that earcons reduced the number of key presses to complete given tasks and also helped the test subjects to complete more tasks successfully.

In [7], Helle et al. have studied the effect and acceptance of a sonified menu structure in a mobile phone. The sonification design was done by using earcons, and largely based on previous studies by Leplatre and Brewster (e.g. [4], [5], and [6]) on user interface sonification in computers and mobile phones. Nokia 8210 mobile phones were used as a test platform, so the implementation had to rely on monophonic buzzer sounds, which were the only available sounds during that time. According to Helle, practically all test participants "considered the sounds disturbing in places where others are present, especially in situations like meetings, but also in public places, trains or buses... About half of the users considered the sounds too long especially in the beginning of the main level." Eleven out of seventeen participants did not find them useful at all.

Luckily, during recent years the audio capabilities of mobile phones have improved greatly. New features like polyphonic sounds and sound effects give new possibilities for designing useful and informative audio feedback for mobile phones.

\subsection{Reverb and Delay}

Reverberation i.e. reverb is probably one of the most heavily used effects in music. It is added on separate instruments and/or the whole mix in order to create a feeling of space. As reverb is always present in our surroundings, a completely dry tone may sound unnatural.

Natural reverberation is the result of the many reflections of a sound that occur in a room or some other space. In addition to the direct sound from a loudspeaker or other sound source, we can also hear the sound waves reflecting from walls, ceiling, floor, and different obstacles in the room. The reflected sound waves arrive to listener's ears a little later than the direct sound, and are generally a little weaker in energy and high frequency content. The delay is so short that each reflection is not perceived as a copy of the original sound. Instead, we hear them as a whole i.e. as a reverb. Reverb can also be generated artificially using a dedicated or some general-purpose effect processor device. 
When the reflections are sparser and can be heard as separate sounds, these sounds are generally known as the 'echo' or 'delay' effect. An artificial delay effect takes in an audio signal and plays it back after the delay time, which can range from several milliseconds to several seconds.

'Reverb time' (often referred to as RT or T60) is the amount of time it takes for the sound pressure level or intensity to decay $60 \mathrm{~dB}$ from its original value. The parameter is usually associated with a room size, and traditional concert halls have reverberation times of about 1.5 to 2 seconds. 'Reverb decay time' (RDT), on the other hand, indicates how long the reverb can be heard after the input sound stops. The definition of 'hearing' varies among manufacturers [8]. Reverb time and reverb decay time are sometimes mixed up in the literature.

Parthy et al. [9] propose "an ambient communication system that modulates the reverberance applied to music with a single variable in order to communicate nonmusical information to the listener." Their test results indicate that human listeners were able to accurately detect changes in RDT from a reference value of two, five, and ten seconds when the RDT increases by more than $60 \%$ or decreases by more than $30 \%$. Subjects were able to detect the difference between RDTs of 2.0s and 5.0s or greater, but there was a $15 \%$ error rate for RDTs of $1.0 \mathrm{~s}$ and below.

There are also many other parameters related to reverb such as early reflections, high-frequency damping, etc. Of them, only a 'wet/dry ratio' is worth mentioning here as it is related to the implementation of our application. The wet/dry ratio refers to the level ratio of original and reverberated signal, and it is a common parameter in commercial effects processors and an audio editing software.

\subsection{Time-Scale Modification}

There are several ways to change the pitch and duration of a digital audio clip. One common technique is changing the sampling rate of the clip, i.e., resampling. Resampling always affects both the pitch and duration simultaneously. Lowering the sampling rate makes the pitch higher and the duration shorter. Correspondingly, raising the sampling rate lowers the pitch and lengthens the duration. The effect is analogous to a record turntable: Raising the sampling rate produces similar effects as spinning the record faster, and lowering it produces effects similar to spinning the record slower. [17]

It is also possible to change only the pitch or duration without affecting the other. Some of these techniques operate in the time domain, others in the frequency domain [18]. A common characteristic for all these techniques is that they work well only when the change in pitch or duration is relatively small.

The implementation complexity of resampling and time- or pitch-preserving algorithms varies widely. There are algorithms that implement all three types of techniques in real time. In general, the resampling techniques require less processing power than the time- or pitch-preserving techniques. However, there are exceptions to this rule. The simplest methods are most suitable to mobile environment where processing power is in short supply. Examples of suitable techniques are low-order Lagrange interpolators for resampling and synchronous overlap-add (SOLA) algorithms for time- or pitch-preserving processing [17] [18]. 


\section{Our Approach}

Enhancing visual presentation with audio can overcome problems that different contexts set for a mobile device and its use. The objective of our study was to use non-speech audio for three different purposes: data auralization, audio feedback for events, and impressing the user.

We decided to use various signal processing techniques to modify a limited set of UI sounds. As the techniques are applicable to any sound, no additional sound design is then needed and the number of UI sounds is minimized.

\subsection{Sonified Calendar Application Prototype}

We selected the mobile calendar application to act as a demonstrator for our design proposals. The calendar is a feature-rich application that can accommodate several design ideas. In addition, as it is a familiar application for many users and they should be able to judge whether the sonification really enhances its usability.

We copied the basic functionality of the mobile calendar to our prototype in order to maintain consistency with the normal calendar application. Users can create calendar entries and reminders, as well as browse calendar content using month, week, or day views (Fig. 1). The application notifies the user with an alarm when an important event is due in the calendar.

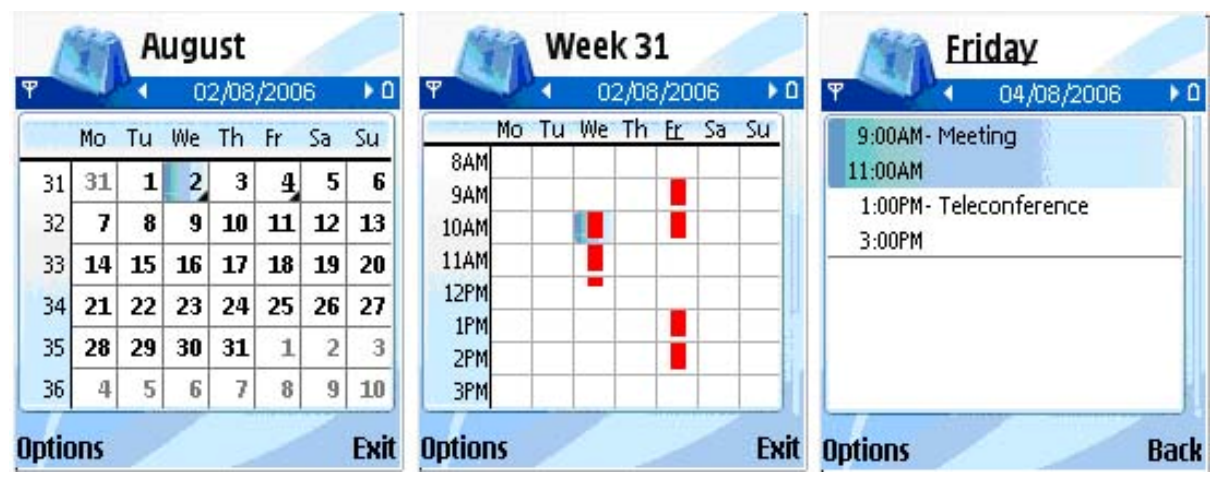

Fig. 1. Basic views in the calendar: month, week, and day views

Calendar entry types are meeting, anniversary, todo, and day note. All entries include title and alarm time. Meeting entries have starting time, duration, and location attributes, and alarm time can be adjusted relative to the meeting starting time.

\subsection{Sonification and Sound Design}

Many previous audio UI designs have been based on auditory icons or earcons. As there was not a clear need to imitate the sounds of real-world objects, we decided not to use auditory icons in our prototype. The main reasons for abandoning earcons were: 
1. The Sonified Calendar does not include complex menu hierarchies that would benefit from the use of earcons;

2. The application was not meant for eyes-free use; and

3. There were studies (e.g. [6] and [7]) indicating that earcons do not work as well in mobile phones as in the PC environment.

Our solution was to select a limited set of simple UI sounds and apply sound effects to them.

In the month view, a short sound is played every time the user moves a cursor between the days. The sound is modified according to the content of the day. If a day has no calendar entries, the sound is played with lots of reverberation to create a feeling of an empty space. Correspondingly, if the day is full of entries, the sound is played without reverberation to create a sensation of the crammed day. There are also some degrees between these two extremes. The level of reverb is adjusted to match the 'fullness' or 'emptiness' of the day, and it decreases as the number of calendar entries increases.

Reverberated sounds were selected by ear i.e. by modifying different parameters and listening to the output. One important criterion was that the sounds could not be too long, as this could slow down the use of the calendar. Also, due to the low quality of mobile phones' loudspeakers, the sounds had to differ quite a lot from each other. The selected sounds with reverb parameters are described in Table 1.

Table 1. Mapping reverb parameters to calendar bookings

\begin{tabular}{|l|l|l|l|}
\hline Sound & $\mathbf{T}_{\mathbf{6 0}}{ }^{2}$ & Wet/Dry Ratio & Calendar Entries \\
\hline 1 & $0 \mathrm{~ms}$ & Dry Sound & 7 hours or more \\
\hline 2 & $700 \mathrm{~ms}$ & $25 \%$ & $5-7$ hours \\
\hline 3 & $1000 \mathrm{~ms}$ & $50 \%$ & $1-5$ hours \\
\hline 4 & $1010 \mathrm{~ms}$ & $100 \%$ & $<1$ hour \\
\hline
\end{tabular}

When the user browses from one month to another, an additional sound is played. This sound has no processing attached to it and it remains always the same.

Each calendar event type has its own sound. The events are visible in the week and day views. When the user navigates to a calendar event, a distinctive UI sound to that type of calendar event is played.

The browsing sounds have also a time-dependent adaptive behavior attached to navigation speed. When the user navigates fast in the views, the browsing sound fades out to the background so that it does not become annoying to the user. When the user navigates slower, the sound becomes more audible again. The application keeps track of time between key presses and adjusts the behavior accordingly. The adaptive fading does not affect the month change sound, which is always played with the same volume level.

Calendar notifications have an alarm sound attached to them. When a notification is triggered, the related alarm sound is also played. It is possible to react to the notification either with 'snooze' or 'dismiss'. Snooze command will postpone the

\footnotetext{
${ }^{2}$ The given $\mathrm{T}_{60}$ times are rough estimates that were measured manually from the waveform.
} 
alarm for five minutes. When the user selects snooze command, a turntable stop effect is applied. The effect sounds like slowing down a vinyl on a turntable. Next time when the alarm is restored, a turntable start effect is applied and the sound restored to its original speed. Dismiss command acknowledges the alarm and a delay effect is applied to the sound. The effect will make the sound to vanish gradually.

\section{Evaluation}

In order to assess the usability of the concept, we arranged a qualitative evaluation.

The purpose of the evaluation was to find out how users perceived audio feedback, and whether it helped them in different tasks and mobile contexts. In addition, we wanted to know what users subjectively thought about the design after a longer period of use. The field trial method was selected because forming an opinion about the audio feedback takes longer than what is normally permitted in a laboratory test. Another reason was that arranging plausible contexts was difficult in a laboratory.

\subsection{Participants}

22 persons participated in a field trial. 55\% of participants were young adults (25-30 years old) while the rest were older. Out of the 22 participants only two were female. We recruited participants by sending an open call for participation email to a couple of big distribution lists within our company. Participants self-registered to the study by sending an email to us. None of the participants had any prior experience with the Sonified Calendar application.

The only criterion for the participants was that they use the mobile phone calendar on a daily basis. Preferably, they should have a calendar in their PC as well, and synchronize it with the mobile phone. Participants represented this target group very well. 17 participants had a calendar in both their mobile device and PC. One participant also used a paper calendar and one participant a PDA calendar. Three participants relied only on the calendar in their mobile device. $82 \%$ of participants synchronized data between their calendars.

It was also presumable that multiple calendar users would have several calendar entries throughout the day. $50 \%$ of our participants reported that days in their calendars are at least partially booked (Fig 2).

\subsection{Apparatus}

When evaluating audio feedback, the sound quality plays a critical role. Participants used their personal mobile devices during the field trial. All devices had similar sound quality. Six participants used a mobile device that has built-in stereo speakers. Three participants had a device, which is specifically designed for playing music.

\subsection{Procedure}

The evaluation was arranged as a field trial, which lasted for one week. We announced a "call for participation" in mailing lists and interested persons could download the application from the web site and install it to their own mobile phone. It 


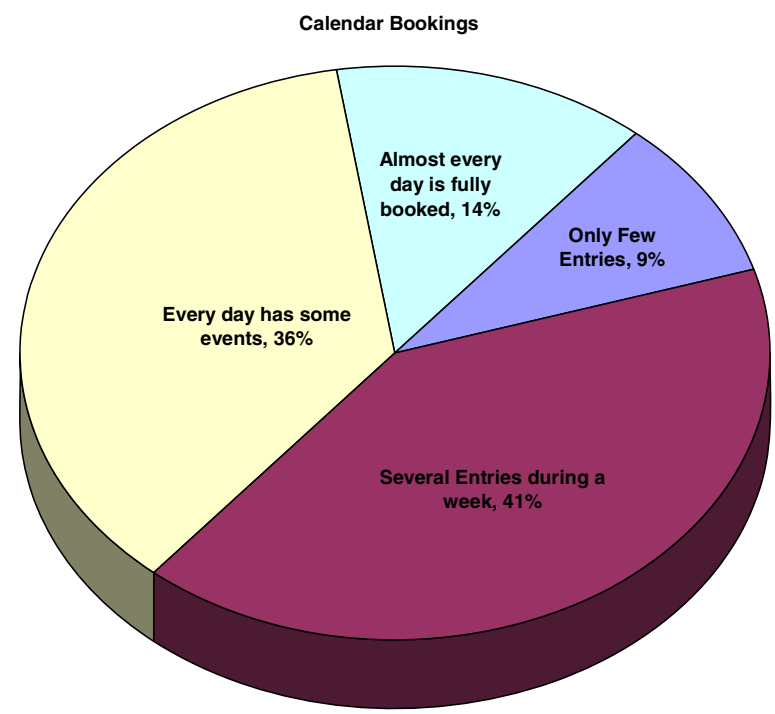

Fig. 2. Level of participants' calendar bookings

was instructed that participants would use our application instead of the original calendar application during the trial. The easiest way to do this was to replace a shortcut in the application menu to our application.

We did not give any specific tasks or situations in which the application should be used. Instead, we encouraged participants to use the application in a similar manner as they would use their current mobile calendar.

After the field trial participants were asked to fill in a web-based questionnaire, which collected subjective opinions about the introduced features.

\section{Results}

Our design goal was that we could introduce features that would be both entertaining and informative for the users. The results indicated that we succeeded with some features, but there is also room for improvement, especially in the sound design.

$64 \%$ of the participants said that our prototype calendar was more informative than the original calendar. In addition, $50 \%$ of the participants said that the prototype was more fun than their old calendar. Finally, $68 \%$ of the participants were interested in hearing similar audio feedback in other applications as well. This is an interesting result because for the evaluation purpose we combined several design ideas into one application, but originally they were designed for various applications.

Despite the fact that participants considered the prototype calendar to be more informative, it was not easier to use. Table 2 illustrates that all features except the month change sound had more merits as an entertaining feature rather than providing information to the user. Sound effects were not really able to provide any navigation aid for the participants. On the other hand, 12 participants found the prototype to be 
more annoying than the normal calendar. There are two reasons for this. Some participants did not like our sound design, while others considered audio feedback in mobile devices altogether annoying.

Table 2. Percentages of participants favoring features as entertaining, informative, or navigation aid

\begin{tabular}{|l|l|l|l|}
\hline Feature & Entertaining & Informative & Useful for navigation \\
\hline Adaptive sound volume & $50 \%$ & $32 \%$ & $23 \%$ \\
\hline Day content auralization & $59 \%$ & $55 \%$ & $23 \%$ \\
\hline Calendar entry sounds & $59 \%$ & $41 \%$ & $45 \%$ \\
\hline Alarms & $59 \%$ & $45 \%$ & NA \\
\hline Month change sound & $68 \%$ & $77 \%$ & $55 \%$ \\
\hline
\end{tabular}

In this study we used non-speech audio for three different purposes: data auralization, feedback for events and actions, and impressing the user. Next we will go through each category and describe how different features succeeded in this study.

\subsection{Data Auralization}

For data auralization we had two ideas implemented in our prototype. The first feature was the use of reverb to illustrate the fullness of the day. This feature was used in the month view, which displays only an indicator if there are events during the day, and more detailed information is available as a tooltip after a short period.

The basic idea of reverb was perceived pretty well and half of the participants found it intuitive. However, the feature turned out to be quite difficult to hear in practice. 12 participants said that they could hear the difference between empty and full days, which have the most noticeable difference in the amount of reverb, but other two levels were more difficult to notice. $45 \%$ of the participants said that they could not really hear other levels even though there were four levels in total. For this reason the majority of participants said that feedback could not be used in navigation. Those participants who could hear the feature, considered it not to be annoying but informative (55\% in favor) and fun (59\% in favor). One reason for this could be that audio feedback was presented faster than the visual feedback, and the participants could at least get a rough estimate if there are any events on the day by hearing the reverberated sound.

The second data auralization feature was that each calendar event had an identifiable sound attached to it. This feature was audible in week and day views and it gave an estimation of what kinds of events the user has marked for the day. This feature was even more difficult to use than the reverb. Only half of the participants noticed that there are different sounds attached to the calendar events. Therefore, the sounds were not very intuitive or informative either. Only $41 \%$ of the participants said that these sounds have some information value. Surprisingly, these sounds were not annoying, but more like fun (59\% in favor). This feature probably requires careful sound design for future iterations. Currently sounds were too similar and many participants said that the sounds were not very good. 


\subsection{Audio Feedback on User's Actions}

In the Sonified Calendar application, there were two new features that provided audio feedback for user's actions. The first one was adaptive sound volume, which lowered the volume of the keypad tones when the participant was actively using the device, and restored the original level after a certain inactivity period. The purpose of this feature was to minimize the disturbance that continuous keypad tones would cause. The keypad tones lose their importance when the user is actively pressing keys, since usually there is also visual feedback available and the user needs to look at the display when completing a task. It can also be quite irritating for other people in the vicinity to listen to continuous click sounds when the user is, for example, typing a long text message.

Half of the participants found the adaptive sound volume feature fun. In addition, $68 \%$ of the participants did not consider it annoying if there are other people in the vicinity. This was actually the main objective of the feature. The device should be able to adapt to the context where it is used and make the usage as pleasant as possible. Participants considered that the feature did not provide much information or help in navigation. On the other hand, the purpose of this feature was not to provide any information, but to demonstrate how the device can adapt to the current interaction that the user has with the device. Overall, participants were quite satisfied with how the feature was implemented. The adaptation rate of volume level seemed to be in balance although some participants said that it could have been slightly more responsive to user's actions. Volume change speed should also be fine-tuned a bit to find the right speed. Adaptive sound volume feature was the only feature in the application in which the average user satisfaction at the end of the study was slightly higher than at the beginning, but the increase was not statistically significant.

The second feature that provided feedback on user's actions was a month change sound. This feature was the simplest audio feedback that we had in our prototype. The participants could hear a sound whenever focus moved to the next or previous month. Even though adaptive volume affected to all other sounds that were used in month, week, and day views, we wanted to keep this sound unaffected. $86 \%$ of the participants noticed the sound when they were browsing days in their calendar, and $77 \%$ of them found it intuitive and informative. Surprisingly, $68 \%$ of the participants found this feature also fun. We are not sure what made this feature fun, but it might have been the sound that we used as a sound clip. 12 participants said that they listened to the sound when they were navigating. This feature was an example of audio feedback that can enhance the usability of a user interface in a very simple manner.

\subsection{Impressing the User}

The most noticeable feature that was implemented intentionally to impress the user and be fun was sound effects that were attached to the calendar alarms. There were three different sound effects: turntable start, turntable stop, and fading delay.

Even though these sound effects were considered to be fun (59\% in favor), they have also information value. $45 \%$ of participants considered the turntable sound effects to be informative, and for the delay effect the information value was slightly 
less. Sound effects confirmed the key presses in a more informative manner than simple keypad tones. Furthermore, applying the turntable start effect at the beginning of the alarm would inform the user that this alarm has been postponed earlier. Participants ranked these sound effects to be the best-loved feature in the application. The average user satisfaction was slightly lower at the end of the study compared to initial impression, but the decrease was not statistically significant.

\subsection{Latency}

Although real-time processing can be a bottleneck in mobile devices due to low processing power, Sonified Calendar's technical implementation seemed to be quite successful. $68 \%$ of the participants said that they did not perceive any latency in sound processing and that the application was not any slower than the original application without any audio feedback. Only three participants said that the application user interface felt slower because of audio, but this can be probably improved by optimizing the implementation. Anyway our application was a prototype and we did not have any strict requirements of minimizing the latency.

\section{Discussion and Future Work}

The usability evaluation of our Sonified Calendar application showed that it is possible to improve the usability of a mobile device by enhancing its visual user interface with audio feedback. Sound effects have two benefits compared to other audio methods. Many users value the possibility to personalize their mobile devices, and sound effects enable them to utilize their favorite sounds in the UI. User interface designers can still design audio feedback as they see it best. Furthermore, sound effects decrease the work load of sound designers, since they do not have to design a new sound for every situation, but can apply different sound effects depending on the case.

The evaluation showed that the selected sound effects are capable of providing information on a general level, but more detailed level may require some visual information or some other kind of audio feedback such as speech.

Another interesting aspect of the evaluation was that participants seemed to like audio feedback and they found many features amusing. This might result from the current usage pattern in which devices are usually used without any sound.

In this study, we demonstrated the benefits of sound effects in one application, but the next step would be to design appropriate audio feedback to other applications as well and to validate them at the same time. The features that we presented in the calendar application were originally developed for different applications like phone calls, photo gallery, and general navigation in the mobile user interface. However, for the evaluation purposes a collection of the features were consolidated into one application in order to make the evaluation more efficient.

Mobile devices are moving towards multimodal user interfaces, which presumable increase the utilization of audio modality. In addition, some information will be presented using haptic modality. The possibilities for combining sound effects and audio in general with haptic feedback should be studied further. 


\section{Conclusions}

We have developed a Sonified Calendar application prototype in order to study how users perceive sound effects as audio feedback in mobile user interfaces. In order to assess the usability of the design, we arranged a field trial that allowed participants to use the application in real mobile context with their own calendar entries. The results indicated that sound effects are capable of transmitting useful information in some extent, but a more likely utilization is in impressing the user and making the mobile user interface more fun.

Acknowledgements. We would like to thank Jussi Sinkkonen, Saara Lehtola, and Jukka Kaartinen for their help in implementing the Sonified Calendar application prototype.

\section{References}

1. Kramer, G., et al.: Sonification Report: Status of the Field and Research Agenda (1997), http://www.icad.org/websiteV2.0/References/nsf.html

2. Hermann, T., Dres, J., Ritter, H.: Broadcasting Auditory Weather Reports - A Pilot Project. In: ICAD. Proceedings of International Conference on Auditory Display (2003)

3. Gaver, W.: Auditory Interfaces. In: Helander, M., Landauer, T., Prabhu, P. (eds.) Handbook of Interaction, Elsevier, Amsterdam (1997)

4. Brewster, S., Wright, P., Edwards, A.: An Evaluation of Earcons for Use in Auditory Human-Computer Interfaces. In: Proceedings of INTERCHI Conference (1993)

5. Brewster, S., Räty, V-P., Kortekangas, A.: Earcons as a Method of Providing Navigational Cues in a Menu Hierarchy. In: Proceedings of HCI Conference (1996)

6. Leplatre, G., Brewster, S.: Designing Non-Speech Sounds to Support Navigation in Mobile Phone Menus. In: ICAD. Proceedings of International Conference on Auditory Display (2000)

7. Helle, S., Leplatre, G., Marila, J., Laine, P.: Menu Sonification in a Mobile Phone - A Prototype Study. In: ICAD. Proceedings of International Conference on Auditory Display (2001)

8. Lehman, S.: Reverberation (accessed April 11th, 2007) (1996), Avalable online at: http://www.harmony-central.com/Effects/Articles/Reverb/

9. Parthy, A., Jin, C., van Schaik, A.: Reverberation for Ambient Data Communication. In: ICAD. Proceedings of International Conference on Auditory Display (2004)

10. Gaver, W., Smith, R., O’Shea, T.: Effective Sounds in Complex Systems: The Arkola Simulation. In: Proceedings of ACM Conference, ACM Press, New York (1991)

11. Larsson, P., Västfjäll, D., Kleiner, M.: Do We Really Live in a Silent World? The (Mis)use of Audio in Virtual Environments. In: Proceedings of AVR II and CONVR Conference, pp. 182-188 (2001)

12. Bly, S.: Presenting Information in Sound. In: Proceedings of Human Factors in Computer Systems, pp. 371-375 (1982)

13. Mezrich, J.J, Frysinger, S., Slivjanovski, R.: Dynamic Representation of Multivariate Time Series Data. Journal of the American Statistical Association 79(385), 34-40 (1984) 
14. Brown, M., Newsome, S., Glinert, E.: An Experiment into the Use of Auditory Cues to Reduce Visual Workload. In: Proceedings of Computer-Human Interaction Conference, pp. 339-346 (1989)

15. Janata, P., Childs, E.: Marketbuzz: Sonification of Real-Time Financial Data. In: ICAD. Proceedings of International Conference on Auditory Display (2004)

16. Dombois, F.: Auditory Seismology: On Free Oscillations, Focal Mechanisms, Explosions, and Synthetic Seismograms. In: ICAD. Proceedings of International Conference on Auditory Display (2002)

17. Holm, J.: Pitch-Shifting Methods for Wavetable Synthesis. M.Sc. Thesis, Tampere University of Technology (2000)

18. Laroche, J.: Time and Pitch Scale Modification of Audio Signals. In: Kahrs, M., Brandenburg, K. (eds.) Applications of Digital Signal Processing to Audio and Acoustics, Kluwer Academic Publishers, Boston, MA (1998)

19. Davies, T.C., Burn, C.M.: Do You Hear What I Hear? Reflecting on Auditory Display in Medicine (accessed April 11th, 2007), Available online at: http://hcro.enigma.co.nz/ website/ index.cfm?fuseaction $=$ articledisplay\&FeatureID $=040906$ 